\title{
MHD SELF-SIMILAR SOLUTIONS FOR COLLIMATED JETS
}

\author{
E. TRUSSONI \\ Osservatorio Astronomico di Torino, Pino T.se, ITALY \\ C. SAUTY
}

Observatoire de Paris, DAEC, Meudon, FRANCE

AND

K. TSINGANOS

University of Crete, FORTH, Heraklion, GREECE

The MHD modelling of jets in axisymmetric geometry requires the treatment of the Bernoulli and the transfield equations, that can be treated following a self-similar approach. This technique is based on two main assumptions: i) the physical variables are factorized; ii) a suitable scaling law in one direction is prescribed. Solutions self-similar in the $r$ direction (in a spherical frame of reference) have been studied to model collimated winds from disks (Blandford and Payne 1982). Here we present solutions self-similar in the $\theta$ direction, suitable to study the collimated wind around the polar axis of a rotating object (Tsinganos and Trussoni 1991, Sauty and Tsinganos 1994). Our basic assumptions are:

- The magnetic flux function, that describes the poloidal components of velocity and magnetic field, is expressed as $A(r, \theta) \propto f(r) \sin ^{2} \theta$.

- The density and the pressure of the plasma are assumed to scale linearly with $A: \rho(r, \theta) \propto 1+\delta A$ and $P(r, \theta) \propto P_{o}(r)(1+K f A)$. Accordingly, the surfaces with equal poloidal Alfvén number $M$ are spherical.

The original MHD equations then reduce to three ordinary differential equations for the four variables $M, f, P_{o}$ and $P_{1}(\equiv K f A)$. To close the system we need further assumptions, that define two classes of solutions:

1) $P_{o}$ and $P_{1}$ are related, i.e. $K f A=\kappa=$ const; the unknown is $f$ and the shape of the streamlines is deduced. For these solutions a characteristic integral exists $(\epsilon)$ : it is $\epsilon>0$ or $\epsilon<0$ whether the volumetric energy along the polar axis is lower or higher than along the fieldlines, respectively.

2) $P_{o}$ and $P_{1}$ are kept unrelated, then the function $f$ must be prescribed. By assuming $f \propto R^{n}$ we can choose streamlines radially expanding $(n=0)$ 

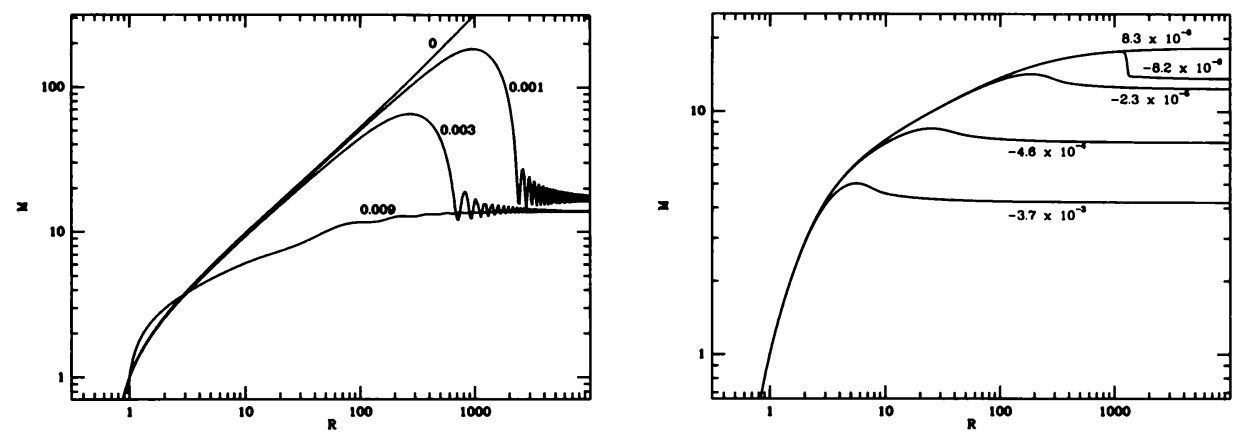

Figure 1. $M$ vs $R$ along the polar axis for solutions of class 1 (left panel), for $\epsilon / \lambda^{2}=-0.2$ and some values of $\kappa / \lambda^{2}$, and class 2 (right panel) for $n=2$ and some values of $q$

or cylindrically collimated $(n=2)$. These solutions are characterized by the parameter $q \propto\left(Q_{1} / r^{2}\right)_{r \rightarrow \infty}$.

Typical results are shown in Fig. 1 for $\lambda=3(\lambda$ is the ratio of the azimuthal to the poloidal velocity at the Alfvèn point). For both kinds of solutions outflows with superAlfvénic velocities and collimated streamlines can be found. In the former case, for $\kappa=0$ collimated flows are found when $\epsilon>0$, while for $\epsilon<0$ the fieldlines are asymptotically radial and $M$ diverges. For $\kappa>0$ collimation is found asymptotically also for $\epsilon<0: M$ has a maximum before attaining a constant terminal value. In the collimated region the Alfvèn number has oscillations with decreasing amplitude, related to periodic pinching of the sectional area of the jet. The other class of solutions depend critically on the value of $n$.

In the case of prescribed streamlines, if they are radially expanding ( $n=$ $0)$ the asymptotic velocity slightly increases $\left[\propto(\log r)^{1 / 3}\right]$ in the asymptotic region. For collimated fieldlines $(n=2$, see Fig. 1) superAlfvénic solutions are found with constant asymptotic velocities for several values of $q$. We notice that again the flow speed can have a maximum before reaching the final velocity, consistently with the behaviour of the other class of solutions.

Our results can be considered complementary with those coming from a $r$ self-similar treatment, not valid on the rotational axis.

\section{References}

Blandford R.D. and Payne D.G., 1982, M.N.R.A.S., 199, 883.

Sauty C. and Tsinganos K., 1994, Astr. Ap., 287, 893.

Tsinganos K. and Trussoni E., 1991, Astr. Ap., 249, 156. 